\title{
INTERACTIVE IMPACTS OF PLANT GROWTH REGULATORS AND FERTIGATION ON QUALITY OF POMEGRANATE (PUNICA GRANATUM L.) IN SUB-HUMID AGRO ECOLOGICAL ZONE OF RAJASTHAN
}

\author{
Mahaveer Suman* AND MC Jain \\ Department of Fruit Science, College of Horticulture and Forestry, \\ Jhalawar, Agriculture University, Kota (Rajasthan) India
}

Keywords: Ethrel, Fertilizers, Fruit quality, NAA, Spray

\begin{abstract}
Individual and interaction effects of plant growth regulators and fertigation on quality of pomegranate were investigated. Maximum mean polar diameter $(9.59 \mathrm{~cm})$, equatorial diameter $(8.10 \mathrm{~cm})$, fruit weight (206.06 g), fruit volume (190.86 cc), number of arils per fruit (416.81), aril weight per fruit (146.35 g), weight of 100 arils (35.86 g) and aril per cent (70.92\%) along with minimum specific gravity (1.090), rind thickness $(5.15 \mathrm{~mm})$, rind per cent $(29.09 \%)$ and fruit cracking $(5.58 \%)$ were recorded in $\mathrm{F}_{2}$ (100 per cent RDF through fertigation). Similarly, spray of $100 \mathrm{ppm}$ NAA $\left(\mathrm{N}_{2}\right)$ and $250 \mathrm{ppm}$ ethrel $\left(\mathrm{E}_{2}\right)$ significantly influenced the above characteristics of fruit. Treatment combination of 100 ppm NAA +150 ppm ethrel $+100 \%$ RDF through fertigation $\left(\mathrm{N}_{2} \mathrm{E}_{1} \mathrm{~F}_{2}\right)$ gave better quality fruit over other treatments including control.
\end{abstract}

\section{Introduction}

Pomegranate (Punica granatum L.) one of the most promising fruit crops of India belongs to Lythraceae. Pomegranate is one of the dollar earning table fruits in the world, for its refreshing juice with nutritional and medicinal properties. Fruit juice is a good source of sugars, vitamin $\mathrm{C}$, vitamin B, pantothenic acid, potassium, antioxidant polyphenols and a fair source of iron. The plant growth regulators have been used for various beneficial effects such as to modify a crop by changing the rate or pattern or both of its response to the internal and external factors that govern development from germination through vegetative growth, reproductive development, maturity and senescence or aging, promoting root growth and the number of flowers, increasing the fruit set, fruit size, quality and for inducing early and uniform fruit ripening as well as postharvest preservation (Mikal 1999). Application of fertilizers through fertigation, improves fertilizer and water use efficiency, helps to maintain nutritional balance and nutrient concentration at optimum level, provides opportunity to apply the nutrients at critical stages of crop growth and minimizes hazard of ground water pollution due to nitrate leaching as compared to conventional practice of fertilizers application (Thiyagarajan 2006). Plant growth regulators and fertigation are the most important inputs which directly affect the plant growth, development, yield and quality of product. Farmers are using solid fertilizers for fruit crop production but these are not totally water soluble and hence are less available to plants and some of the fertilizers contain salts of sodium and chloride which not only affect the quality and quantity of crop production but they are also harmful to the soil. Keeping these facts in view the present experiment was carried out.

\section{Materials and Methods}

The present investigation was carried out in the Department of Fruit Science, College of Horticulture and Forestry, Jhalawar, Agriculture University, Kota. The experiment was conducted in the pomegranate orchard established under high density planting system $(3 \mathrm{~m} \times 3 \mathrm{~m})$ at the Krishi Vigyan Kendra, Jhalawar in the near vicinity of the college during July 2018 to December

*Author for correspondence: <mahaveersuman001@ gmail.com>. 
2018 and again during July 2019 to December 2019. Six years old pomegranate plants of uniform size and growth were selected at the Krishi Vigyan Kendra, Jhalawar, (Agriculture University, Kota) for experimentation. The experiment was laid out in Factorial Randomized Block Design and each treatment was replicated thrice and per treatment two plants were used. The experiment comprised of 27 treatment combinations consisting of plant growth regulators levels (NAA 0, 50 and $100 \mathrm{ppm}$ and ethrel 0,150 and $250 \mathrm{ppm})$ and fertigation levels $(0,75$ and $100 \%$ recommended dose of fertilizers). The plant growth regulators were sprayed at pre flowering and post flowering stage. The fertigation schedule (625:250:250 g/plant/year) for six years old pomegranate plants was fixed based on the recommendation given by Pareek (1982). For fertigation, the above fertilizers dose were divided in four equal split dose and applied monthly from 1 July to 1 October on both years. Diameter and rind thickness of fruit in each treatment was recorded with the help of digital vernier caliper, average fruit weight, aril weight, weight of 100-arils and rind weight were recorded with the help of electronic balance. Fruit volume was measured by the water displacement method, specific gravity of the fruits was worked out by dividing the weight of fruit by volume of same fruit, aril per cent and rind per cent was calculated by the total aril weight and rind weight divided by the fruit weight and multiply by hundred, respectively.

\section{Result and Discussion}

Among the various levels of NAA treatment, application of $100 \mathrm{ppm}$ NAA $\left(\mathrm{N}_{2}\right)$ showed maximum mean polar diameter $(9.57 \mathrm{~cm})$,equatorial diameter $(8.05 \mathrm{~cm})$ and fruit weight $(203.95$ $\mathrm{g})$. In the ethrel treatment maximum mean polar diameter $(9.31 \mathrm{~cm})$, equatorial diameter $(7.80 \mathrm{~cm})$ and fruit weight $(198.77 \mathrm{~g})$ were observed under the ethrel $250 \mathrm{ppm}\left(\mathrm{E}_{2}\right)$. Under the different levels of fertigation treatments, maximum mean polar diameter $(9.59 \mathrm{~cm})$, equatorial diameter $(8.10 \mathrm{~cm})$ and fruit weight $(206.06 \mathrm{~g})$ were noticed in $\left(\mathrm{F}_{2}\right) 100$ per cent RDF through fertigation (Table 1). Further, treatment combination of NAA $100 \mathrm{ppm}+$ ethrel $150 \mathrm{ppm}+$ fertigation $100 \%$ $\mathrm{RDF}\left(\mathrm{N}_{2} \mathrm{E}_{1} \mathrm{~F}_{2}\right)$ was recorded maximum mean polar diameter $(10.65 \mathrm{~cm})$, equatorial diameter $(9.21$ $\mathrm{cm})$ and fruit weight (227.57 g) as compared to other treatments including control (Table 2). Increase in fruit size with the application of NAA could be due to nature of auxins to stimulate cell division and cell enlargement and increase sink strength of the fruits (Thakur and Sharma 2018). On the other hand, better response of fertigation on fruit diameter might be due to uninterrupted supply of major nutrients by fertigation till fruit enlargement stage that resulted in upholding of high nutrients availability during the crop hence, encouraging superior fruit size. The increased fruit weight might be due to the balanced availability of macronutrients. The balanced uptake of nutrients might help the better metabolic activities in the plant ultimately leading to high protein and carbohydrate synthesis (Sinha et al. 2019).

Due to $100 \mathrm{ppm}$ NAA $\left(\mathrm{N}_{2}\right)$ the highest response on fruit volume $(188.78 \mathrm{cc})$, number of arils per fruit (412.59), aril weight per fruit $(144.61 \mathrm{~g})$, weight of 100 arils $(35.73 \mathrm{~g})$ and aril per cent $(70.68 \%)$ were recorded. Similarly, $250 \mathrm{ppm}$ ethrel $\left(\mathrm{E}_{2}\right)$ confer highest number of arils per fruit (404.87), aril weight per fruit (138.70 g), weight of 100-arils (34.85 g) and aril per cent (69.59\%). Furthermore, maximum fruit volume $(182.32 \mathrm{cc})$ observed in the treatment $\mathrm{E}_{1}$, consisted of 150 $\mathrm{ppm}$ ethrel. In the fertigation treatment, maximum fruit volume $(190.86 \mathrm{cc})$, number of arils per fruit (416.81), aril weight per fruit (146.35 g), weight of 100 arils $(35.86 \mathrm{~g})$ and aril per cent $(70.92 \%)$ were noticed in $\left(\mathrm{F}_{2}\right) 100$ per cent RDF through fertigation (Tables 1 and 3). Further, in interaction effect spray of plant growth regulators and fertigation were found to be quite superior than their individual effect. Among the treatment combinations $\mathrm{N}_{2} \mathrm{E}_{1} \mathrm{~F}_{2}$ (NAA $100 \mathrm{ppm}+$ ethrel $150 \mathrm{ppm}+$ fertigation $100 \% \mathrm{RDF}$ ) exhibited significantly higher values of fruit volume (217.27 cc), number of arils per fruit (443.26), aril weight per fruit (170.56 g), weight of 100-arils (38.99 g) and aril per cent $(74.91 \%)$ as compared to other treatments including control (Tables 2 and 
Table 1. Effect of plant growth regulators and fertigation on fruit diameter $(\mathrm{cm})$, fruit weight $(\mathrm{g})$ fruit volume (cc), specific gravity and number of arils per fruit of pomegranate under high density planting system.

\begin{tabular}{|c|c|c|c|c|c|c|c|c|c|}
\hline \multirow[t]{2}{*}{ Treatments } & \multicolumn{3}{|c|}{ Polar diameter $(\mathrm{cm})$} & \multicolumn{3}{|c|}{ Equatorial diameter $(\mathrm{cm})$} & \multicolumn{3}{|c|}{ Fruit weight $(\mathrm{g})$} \\
\hline & 2018 & 2019 & Pooled & 2018 & 2019 & Pooled & 2018 & 2019 & Pooled \\
\hline \multicolumn{10}{|c|}{ NAA $(\mathrm{N})$} \\
\hline $\mathrm{N}_{0}$ & 8.45 & 8.76 & 8.60 & 7.03 & 7.22 & 7.13 & 184.25 & 186.50 & 185.37 \\
\hline $\mathrm{N}_{1}$ & 9.06 & 9.37 & 9.21 & 7.65 & 7.77 & 7.71 & 196.21 & 198.32 & 197.28 \\
\hline $\mathrm{N}_{2}$ & 9.36 & 9.78 & 9.57 & 7.97 & 8.13 & 8.05 & 202.57 & 205.33 & 203.95 \\
\hline $\mathrm{SEm} \pm$ & 0.04 & 0.05 & 0.03 & 0.04 & 0.04 & 0.04 & 0.51 & 0.63 & 0.37 \\
\hline C.D. at $5 \%$ & 0.12 & 0.15 & 0.10 & 0.11 & 0.12 & 0.12 & 1.46 & 1.77 & 1.06 \\
\hline \multicolumn{10}{|c|}{ Ethrel (E) } \\
\hline $\mathrm{E}_{0}$ & 8.66 & 8.92 & 8.79 & 7.24 & 7.36 & 7.30 & 188.24 & 190.37 & 189.32 \\
\hline $\mathrm{E}_{1}$ & 9.10 & 9.48 & 9.29 & 7.71 & 7.87 & 7.79 & 197.22 & 199.81 & 198.52 \\
\hline $\mathrm{E}_{2}$ & 9.11 & 9.51 & 9.31 & 7.72 & 7.88 & 7.80 & 197.56 & 199.98 & 198.77 \\
\hline $\mathrm{SEm} \pm$ & 0.04 & 0.05 & 0.03 & 0.04 & 0.04 & 0.04 & 0.51 & 0.625 & 0.37 \\
\hline C.D. at $5 \%$ & 0.12 & 0.15 & 0.10 & 0.11 & 0.12 & 0.12 & 1.46 & 1.77 & 1.06 \\
\hline \multicolumn{10}{|c|}{ Fertigation (F) } \\
\hline $\mathrm{F}_{0}$ & 8.23 & 8.51 & 8.37 & 6.80 & 6.94 & 6.87 & 176.77 & 179.46 & 178.12 \\
\hline $\mathrm{F}_{1}$ & 9.26 & 9.61 & 9.43 & 7.85 & 7.99 & 7.92 & 201.45 & 203.35 & 202.42 \\
\hline $\mathrm{F}_{2}$ & 9.39 & 9.79 & 9.59 & 8.00 & 8.18 & 8.10 & 204.79 & 207.34 & 206.06 \\
\hline $\mathrm{SEm} \pm$ & 0.04 & 0.05 & 0.03 & 0.04 & 0.04 & 0.04 & 0.51 & 0.63 & 0.37 \\
\hline C.D. at $5 \%$ & 0.12 & 0.15 & 0.10 & 0.11 & 0.12 & 0.12 & 1.46 & 1.77 & 1.06 \\
\hline \multirow[t]{3}{*}{ Treatments } & \multicolumn{3}{|c|}{ Fruit volume (cc) } & \multicolumn{3}{|c|}{ Specific gravity } & \multicolumn{3}{|c|}{ Number of arils per fruit } \\
\hline & 2018 & 2019 & Pooled & 2018 & 2019 & Pooled & 2018 & 2019 & Pooled \\
\hline & & & & \multicolumn{3}{|c|}{ NAA $(\mathrm{N})$} & & & \\
\hline $\mathrm{N}_{0}$ & 166.49 & 166.25 & 166.37 & 1.131 & 1.124 & 1.128 & 380.67 & 385.31 & 382.99 \\
\hline $\mathrm{N}_{1}$ & 182.38 & 180.59 & 181.49 & 1.104 & 1.101 & 1.106 & 400.94 & 406.42 & 403.68 \\
\hline $\mathrm{N}_{2}$ & 188.35 & 189.21 & 188.78 & 1.095 & 1.089 & 1.092 & 409.47 & 415.70 & 412.59 \\
\hline $\mathrm{SEm} \pm$ & 0.59 & 0.71 & 0.51 & 0.001 & 0.001 & 0.001 & 0.56 & 0.39 & 0.54 \\
\hline C.D. at $5 \%$ & 1.66 & 2.01 & 1.46 & 0.003 & 0.004 & 0.003 & 1.58 & 1.10 & 1.54 \\
\hline \multicolumn{10}{|c|}{ Ethrel (E) } \\
\hline $\mathrm{E}_{0}$ & 173.88 & 171.10 & 172.49 & 1.116 & 1.115 & 1.120 & 388.04 & 392.65 & 390.34 \\
\hline $\mathrm{E}_{1}$ & 182.42 & 182.21 & 182.32 & 1.108 & 1.101 & 1.105 & 401.28 & 406.82 & 404.05 \\
\hline $\mathrm{E}_{2}$ & 180.93 & 182.74 & 181.83 & 1.105 & 1.098 & 1.102 & 401.77 & 407.96 & 404.87 \\
\hline $\mathrm{SEm} \pm$ & 0.59 & 0.71 & 0.51 & 0.001 & 0.001 & 0.001 & 0.56 & 0.39 & 0.54 \\
\hline C.D. at $5 \%$ & 1.66 & 2.01 & 1.46 & 0.003 & 0.004 & 0.003 & 1.58 & 1.10 & 1.54 \\
\hline \multicolumn{10}{|c|}{ Fertigation (F) } \\
\hline $\mathrm{F}_{0}$ & 159.78 & 158.04 & 158.91 & 1.146 & 1.136 & 1.141 & 367.90 & 371.99 & 369.95 \\
\hline $\mathrm{F}_{1}$ & 187.06 & 186.67 & 186.86 & 1.096 & 1.096 & 1.096 & 409.45 & 415.56 & 412.50 \\
\hline $\mathrm{F}_{2}$ & 190.39 & 191.34 & 190.86 & 1.093 & 1.086 & 1.090 & 413.74 & 419.88 & 416.81 \\
\hline $\mathrm{SEm} \pm$ & 0.59 & 0.71 & 0.51 & 0.001 & 0.001 & 0.001 & 0.56 & 0.39 & 0.54 \\
\hline C.D. at $5 \%$ & 1.66 & 2.01 & 1.46 & 0.003 & 0.004 & 0.003 & 1.58 & 1.10 & 1.54 \\
\hline
\end{tabular}

$\mathrm{N}_{0}$ - NAA 0 ppm, $\mathrm{E}_{0}-$ Ethrel0 ppm, $\mathrm{F}_{0}-$ RDF 0\% Fertigation, $\mathrm{N}_{1}-\mathrm{NAA} 50 \mathrm{ppm}, \mathrm{E}_{1}-$ Ethrel150 ppm, $\mathrm{F}_{1}-$ RDF $75 \%$ Fertigation, $\mathrm{N}_{2}-$ NAA 100 ppm, $\mathrm{E}_{2}-$ Ethrel250 ppm, $\mathrm{F}_{2}$ - RDF 100\% Fertigation.

4).Volume of the fruit showed the same trend as it was observed in case of average weight of fruit. There is a direct relationship between volume of fruit and weight of fruit in most of the fruit crops.The weight of arils is the second important character which contributes to the weight of fruits. The optimum moisture and nutrients directly to the root zone area increased the aril weight 
Table 2. Interaction effect of plant growth regulators and fertigation on fruit diameter $(\mathrm{cm})$, fruit weight $(\mathrm{g})$ and fruit volume of pomegranate under high density planting system.

\begin{tabular}{|c|c|c|c|c|c|c|c|c|c|c|c|c|}
\hline \multirow[t]{2}{*}{ Tr. } & \multicolumn{3}{|c|}{ Polar diameter $(\mathrm{cm})$} & \multicolumn{3}{|c|}{ Equatorial diam. $(\mathrm{cm})$} & \multicolumn{3}{|c|}{ Fruit weight (g) } & \multicolumn{3}{|c|}{ Fruit volume (cc) } \\
\hline & 2018 & 2019 & Pooled & 2018 & 2019 & Pooled & 2018 & 2019 & Pooled & 2018 & 2019 & Pooled \\
\hline $\mathrm{N}_{0} \mathrm{E}_{0} \mathrm{~F}_{0}$ & 7.86 & 8.08 & 97 & 6.22 & 6.40 & 6.31 & 168.25 & 170.32 & 169.28 & 149.19 & 147.33 & 148.26 \\
\hline $\mathrm{N}_{0} \mathrm{E}_{0} \mathrm{~F}_{1}$ & 8.46 & 8.80 & 8.63 & 7.06 & 7.20 & 13 & 187.26 & 188.69 & 187.98 & 168.23 & 168.03 & 168.13 \\
\hline $\mathrm{N}_{0} \mathrm{E}_{0} \mathrm{~F}_{2}$ & 8.55 & 8.9 & 8.73 & 7.15 & 7.22 & & 95 & 190.44 & 20 & 170.62 & 170.91 & 0.76 \\
\hline $\mathrm{N}_{0} \mathrm{E}_{1} \mathrm{~F}_{0}$ & 8.12 & 8.27 & 20 & 6.72 & 7.06 & 6.89 & 173.27 & 175.64 & 174.45 & 154.25 & 152.64 & 153.44 \\
\hline $\mathrm{N}_{0} \mathrm{E}_{2} \mathrm{~F}_{0}$ & 8.17 & 8.45 & 8.31 & 6.77 & 7.10 & 93 & 173.57 & 176.82 & 175.19 & 155.92 & 155.09 & 155.51 \\
\hline $\mathrm{N}_{1} \mathrm{E}_{0} \mathrm{~F}_{0}$ & 8.25 & 8.52 & 8.39 & 6.84 & 6.85 & 6.84 & 175.24 & 178.87 & 177.05 & 160.57 & 157.51 & 159.04 \\
\hline $\mathrm{N}_{2} \mathrm{E}_{0} \mathrm{~F}_{0}$ & 8.28 & 8.56 & 8.42 & 6.88 & 6.91 & 6.89 & 175.89 & 179.32 & 177.60 & 158.55 & 158.18 & 158.36 \\
\hline $\mathrm{N}_{0} \mathrm{E}_{1} \mathrm{~F}_{1}$ & 8.65 & 8.89 & 77 & 7.25 & 7.37 & 30 & 188.55 & 192.48 & 190.51 & 173.88 & 173.42 & 173.65 \\
\hline $\mathrm{N}_{0} \mathrm{E}_{1} \mathrm{~F}_{2}$ & 8.68 & 9.10 & 8.89 & 7.28 & 7.4 & 7 & 191.18 & 193.67 & 192.43 & 176.51 & 17 & 175.75 \\
\hline $\mathrm{N}_{0} \mathrm{E}_{2} \mathrm{~F}_{1}$ & 8.76 & 9.1 & 8.93 & 7.36 & 7.53 & 44 & 193.60 & 194.62 & 19 & 174.54 & 17 & 5.34 \\
\hline $\mathrm{N}_{0} \mathrm{E}_{2} \mathrm{~F}_{2}$ & 8.84 & 9.19 & 9. & 7.44 & 7.55 & 7.49 & 194.58 & 195.86 & 195.22 & 175.25 & 17 & 176.45 \\
\hline $\mathrm{N}_{1} \mathrm{E}_{1} \mathrm{~F}_{0}$ & 8.30 & 8.64 & 8.47 & 6.90 & 6.93 & 6.92 & 179.28 & 179.67 & 179.47 & 162.26 & 159.00 & 160.63 \\
\hline $\mathrm{N}_{1} \mathrm{E}_{2} \mathrm{~F}_{0}$ & 8.32 & 8.67 & 8.50 & 6.91 & 6.96 & 6.94 & 179.92 & 181.70 & 180.81 & 163.75 & 160.91 & 162.33 \\
\hline $\mathrm{N}_{1} \mathrm{E}_{0} \mathrm{~F}_{1}$ & 8.99 & 9.18 & 9.0 & 7.58 & 7.74 & 66 & 196.23 & 196.34 & 196.29 & 188.24 & 178.88 & 183.56 \\
\hline $\mathrm{N}_{1} \mathrm{E}_{0} \mathrm{~F}_{2}$ & 9.07 & 9.1 & 9. & 7.67 & 7.85 & & 198 & 199 & 19 & 18 & 18 & 185.67 \\
\hline $\mathrm{N}_{2} \mathrm{E}_{1} \mathrm{~F}_{0}$ & 8.41 & 8.71 & 8.5 & 7.01 & 7.16 & 0 & 183.96 & 187.45 & 185.70 & 166.93 & 166.78 & 166.86 \\
\hline $\mathrm{N}_{2} \mathrm{E}_{2} \mathrm{~F}_{0}$ & 8.36 & 8.69 & 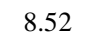 & 6.96 & 7.13 & 7.05 & 181.61 & 185.39 & 18 & 166.59 & 16 & 165.77 \\
\hline $\mathrm{N}_{2} \mathrm{E}_{0} \mathrm{~F}_{1}$ & 9.20 & 9.39 & 9.29 & 7.80 & 7.91 & 7.86 & 200.54 & 201.80 & 201.17 & 189.87 & 185.06 & 187.47 \\
\hline $\mathrm{N}_{2} \mathrm{E}_{0} \mathrm{~F}_{2}$ & 9.32 & 9.68 & 9.50 & 7.92 & 8.07 & 8.00 & 203.88 & 207.89 & 205.88 & 190.83 & 191.54 & 191.18 \\
\hline $\mathrm{N}_{1} \mathrm{E}_{1} \mathrm{~F}_{1}$ & 9.43 & $9 .^{7}$ & 9 & 8.03 & 8 & 8.09 & 20 & 208 & 00 & 19 & 19 & 192.12 \\
\hline $\mathrm{N}_{1} \mathrm{E}_{2} \mathrm{~F}_{2}$ & 9.47 & 9.83 & & 8.07 & 8.2 & & 208.57 & 211.84 & 210.21 & 193.23 & 195.63 & 194.43 \\
\hline $\mathrm{N}_{1} \mathrm{E}_{1} \mathrm{~F}_{2}$ & 9.95 & 10.44 & 10.19 & 8.55 & 8.72 & 8.63 & 212.21 & 215.83 & 214.02 & 198.18 & 201.37 & 199.77 \\
\hline $\mathrm{N}_{1} \mathrm{E}_{2} \mathrm{~F}_{1}$ & 9.73 & 10.14 & 9.93 & 8.33 & 8.54 & 8.44 & 209.66 & 212.86 & 211.26 & 193.51 & 198.15 & 195.83 \\
\hline $\mathrm{N}_{2} \mathrm{E}_{1} \mathrm{~F}_{1}$ & 10.00 & 10.56 & 10.28 & 8.59 & 8.73 & 8.66 & 214.72 & 216.28 & 215.50 & 200.06 & 202.61 & 201.34 \\
\hline $\mathrm{N}_{2} \mathrm{E}_{2} \mathrm{~F}_{2}$ & 10.26 & 10.79 & 10.52 & 8.86 & 9.14 & 9.00 & 219.89 & 221.70 & 220.79 & 203.22 & 209.75 & 206.48 \\
\hline $\mathrm{N}_{2} \mathrm{E}_{1} \mathrm{~F}_{2}$ & 10.34 & 10.97 & 10.65 & 9.10 & 9.32 & 9.21 & 225.96 & 229.17 & 227.57 & 216.82 & 217.71 & 217.27 \\
\hline $\mathrm{N}_{2} \mathrm{E}_{2} \mathrm{~F}_{1}$ & 10.10 & 10.71 & 10.40 & 8.63 & 8.77 & 8.70 & 216.65 & 219.00 & 217.82 & 202.32 & 206.33 & 204.33 \\
\hline $\mathrm{SEm} \pm$ & 0.13 & 0.15 & 0.10 & 0.12 & 0.12 & 0.13 & 1.54 & 1.88 & 1.12 & 1.76 & 2.12 & 1.54 \\
\hline C Dat $5 \%$ & 0.36 & 0.44 & 0.29 & 0.34 & 0.35 & 0.36 & 4.38 & 5.32 & 3.17 & 4.99 & 6.02 & 4.37 \\
\hline
\end{tabular}

$\mathrm{N}_{0}$ - NAA 0 ppm, $\mathrm{E}_{0}-$ Ethrel0 ppm, $\mathrm{F}_{0}-$ RDF 0\% Fertigation, $\mathrm{N}_{1}-\mathrm{NAA} 50 \mathrm{ppm}, \mathrm{E}_{1}-$ Ethrel150 ppm, $\mathrm{F}_{1}-$ RDF $75 \%$ Fertigation, $\mathrm{N}_{2}-$ NAA 100 ppm, $\mathrm{E}_{2}-$ Ethrel $250 \mathrm{ppm}, \mathrm{F}_{2}-$ RDF $100 \%$ Fertigation.

indicating that NPK is essential for grain size and weight. Similar result was reported on aril attributes in pomegranate by Bankar et al.(1990). However, increase in aril weight and aril percentage due to NAA might be attributed to the increase of cell size and intercellular spaces coupled with accumulation of water, sugars and other soluble solids in greater amount as a result of translocation of metabolites (Thakur and Sharma 2018). Nonetheless, increased number of arils by the application of NAA might be due to synthesis of metabolites, increased absorption of water and mobilization of sugars and minerals in the expanded cells and intercellular space which increased the physiological activities leading to increase aril to rind ratio in fruits. The similar results on aril attributes by theapplication of NAA and ethrel treatments in pomegranate was also observed by Shanmugasundaram and Balakrishnamurthy (2017)and Gaikwad et al.(2019). 
Table 3. Effect of plant growth regulators and fertigation on rind weight $(\mathrm{g})$, rind thickness $(\mathrm{mm})$, rind per cent fruit cracking per cent, aril weight per fruit, weight of 100 arils and aril per cent of pomegranate under high density planting system.

\begin{tabular}{|c|c|c|c|c|c|c|c|c|c|c|c|c|}
\hline \multirow[t]{2}{*}{ Tr. } & \multicolumn{3}{|c|}{ Rind weight (g) } & \multicolumn{3}{|c|}{ Rind thickness (mm) } & \multicolumn{3}{|c|}{ Rind per cent } & \multicolumn{3}{|c|}{ Fruit cracking per cent } \\
\hline & 2018 & 2019 & Pooled & 2018 & 2019 & Pooled & 2018 & 2019 & Pooled & 2018 & 2019 & Pooled \\
\hline \multicolumn{13}{|c|}{ NAA (N) } \\
\hline $\mathrm{N}_{0}$ & 63.42 & 61.79 & 62.60 & 5.81 & 5.05 & 5.43 & 34.59 & 33.29 & 33.94 & 6.56 & 5.76 & 6.16 \\
\hline $\mathrm{N}_{1}$ & 60.49 & 58.47 & 59.48 & 5.60 & 4.86 & 5.23 & 30.95 & 29.61 & 30.29 & 6.22 & 5.43 & 5.83 \\
\hline $\mathrm{N}_{2}$ & 60.59 & 58.10 & 59.34 & 5.54 & 4.81 & 5.17 & 30.10 & 28.54 & 29.32 & 6.13 & 5.24 & 5.69 \\
\hline $\mathrm{SEm} \pm$ & 0.26 & 0.31 & 0.20 & 0.02 & 0.02 & 0.02 & 0.22 & 0.24 & 0.16 & 0.01 & 0.03 & 0.02 \\
\hline C.D. at $5 \%$ & 0.75 & 0.88 & 0.55 & 0.07 & 0.06 & 0.05 & 0.61 & 0.69 & 0.45 & 0.03 & 0.09 & 0.06 \\
\hline \multicolumn{13}{|c|}{ Ethrel (E) } \\
\hline $\mathrm{E}_{0}$ & 62.07 & 60.04 & 61.06 & 5.77 & 5.01 & 5.39 & 33.19 & 31.76 & 32.49 & 6.48 & 5.67 & 6.07 \\
\hline $\mathrm{E}_{1}$ & 61.22 & 59.37 & 60.30 & 5.59 & 4.86 & 5.23 & 31.30 & 30.01 & 30.66 & 6.23 & 5.38 & 5.80 \\
\hline $\mathrm{E}_{2}$ & 61.20 & 58.94 & 60.07 & 5.59 & 4.85 & 5.22 & 31.16 & 29.67 & 30.41 & 6.20 & 5.38 & 5.79 \\
\hline $\mathrm{SEm} \pm$ & 0.26 & 0.31 & 0.20 & 0.02 & 0.02 & 0.02 & 0.22 & 0.24 & 0.16 & 0.01 & 0.03 & 0.02 \\
\hline C.D. at $5 \%$ & 0.75 & 0.88 & 0.55 & 0.07 & 0.06 & 0.05 & 0.61 & 0.69 & 0.45 & 0.03 & 0.09 & 0.06 \\
\hline \multicolumn{13}{|c|}{ Fertigation $(\mathrm{F})$} \\
\hline $\mathrm{F}_{0}$ & 62.68 & 61.59 & 62.13 & 5.88 & 5.14 & 5.51 & 35.52 & 34.38 & 34.95 & 6.80 & 6.04 & 6.42 \\
\hline $\mathrm{F}_{1}$ & 61.15 & 58.02 & 59.58 & 5.55 & 4.80 & 5.17 & 30.43 & 28.60 & 29.52 & 6.08 & 5.25 & 5.66 \\
\hline $\mathrm{F}_{2}$ & 60.67 & 58.75 & 59.71 & 5.52 & 4.78 & 5.15 & 29.70 & 28.47 & 29.09 & 6.02 & 5.14 & 5.58 \\
\hline $\mathrm{SEm}+$ & 0.26 & 0.31 & 0.20 & 0.02 & 0.02 & 0.02 & 0.22 & 0.24 & 0.16 & 0.01 & 0.03 & 0.02 \\
\hline C.D. at $5 \%$ & 0.75 & 0.88 & 0.55 & 0.07 & 0.06 & 0.05 & 0.61 & 0.69 & 0.45 & 0.03 & 0.09 & 0.06 \\
\hline \multirow[t]{2}{*}{ Tr. } & \multicolumn{4}{|c|}{ Aril weight per fruit $(\mathrm{g})$} & \multicolumn{4}{|c|}{ Weight of 100 arils (g) } & \multicolumn{4}{|c|}{ Aril per cent } \\
\hline & 2018 & \multicolumn{2}{|c|}{2019} & Pooled & 201 & & 2019 & Pooled & 2018 & 2019 & Pool & \\
\hline \multicolumn{13}{|c|}{ NAA } \\
\hline $\mathrm{N}_{0}$ & 120.82 & & & 122.77 & 32.3 & & 32.77 & 32.56 & 65.41 & 66.71 & 66.0 & \\
\hline $\mathrm{N}_{1}$ & 135.76 & & & 137.80 & 34. & & 34.91 & 34.68 & 69.05 & 70.37 & 69.7 & \\
\hline $\mathrm{N}_{2}$ & 141.98 & & & 144.61 & 35. & & 36.34 & 35.73 & 69.90 & 71.46 & 70.6 & \\
\hline $\mathrm{SEm} \pm$ & 0.35 & & & 0.41 & 0.0 & & 0.13 & 0.09 & 0.23 & 0.23 & 0.1 & \\
\hline C.D. at $5 \%$ & 1.00 & & & 1.15 & 0.2 & & 0.38 & 0.24 & 0.64 & 0.67 & 0.4 & \\
\hline \multicolumn{13}{|c|}{ Ethrel } \\
\hline $\mathrm{N}_{0}$ & 126.20 & & & 128.26 & 33.0 & & 33.76 & 33.42 & 66.81 & 68.22 & 67.5 & \\
\hline $\mathrm{N}_{1}$ & 136.00 & & .44 & 138.22 & 34.3 & & 35.05 & 34.70 & 68.70 & 69.99 & 69.3 & \\
\hline $\mathrm{N}_{2}$ & 136.36 & & & 138.70 & 34.4 & & 35.21 & 34.85 & 68.84 & 70.33 & 69.5 & \\
\hline $\mathrm{SEm} \pm$ & 0.35 & & & 0.41 & 0.0 & & 0.13 & 0.09 & 0.23 & 0.23 & 0.1 & \\
\hline C.D. at $5 \%$ & 1.00 & & & 1.15 & 0.2 & & 0.38 & 0.24 & 0.64 & 0.67 & 0.4 & \\
\hline \multicolumn{13}{|c|}{ Fertigation } \\
\hline $\mathrm{N}_{0}$ & 114.09 & & .87 & 115.98 & 31 & & 31.95 & 31.82 & 64.48 & 65.62 & 65.0 & \\
\hline $\mathrm{N}_{1}$ & 140.34 & & .34 & 142.84 & 34 & & 35.68 & 35.28 & 69.58 & 71.38 & 70.4 & \\
\hline $\mathrm{N}_{2}$ & 144.12 & & .59 & 146.35 & 35 & & 36.39 & 35.86 & 70.30 & 71.53 & 70.9 & \\
\hline $\mathrm{SEm} \pm$ & 0.35 & & & 0.41 & 0. & & 0.13 & 0.09 & 0.23 & 0.23 & 0.15 & \\
\hline C.D. at $5 \%$ & 1.00 & & & 1.15 & 0. & & 0.38 & 0.24 & 0.64 & 0.67 & 0.44 & \\
\hline
\end{tabular}

$\mathrm{N}_{0}$ - NAA 0 ppm, $\mathrm{E}_{0}-$ Ethrel0 ppm, $\mathrm{F}_{0}-\mathrm{RDF} 0 \%$ Fertigation, $\mathrm{N}_{1}-\mathrm{NAA} 50 \mathrm{ppm}, \mathrm{E}_{1}-$ Ethrel150 ppm, $\mathrm{F}_{1}-\mathrm{RDF} 75 \%$ Fertigation, $\mathrm{N}_{2}-\mathrm{NAA} 100 \mathrm{ppm}, \mathrm{E}_{2}-$ Ethrel250 ppm, $\mathrm{F}_{2}-$ RDF $100 \%$ Fertigation. 


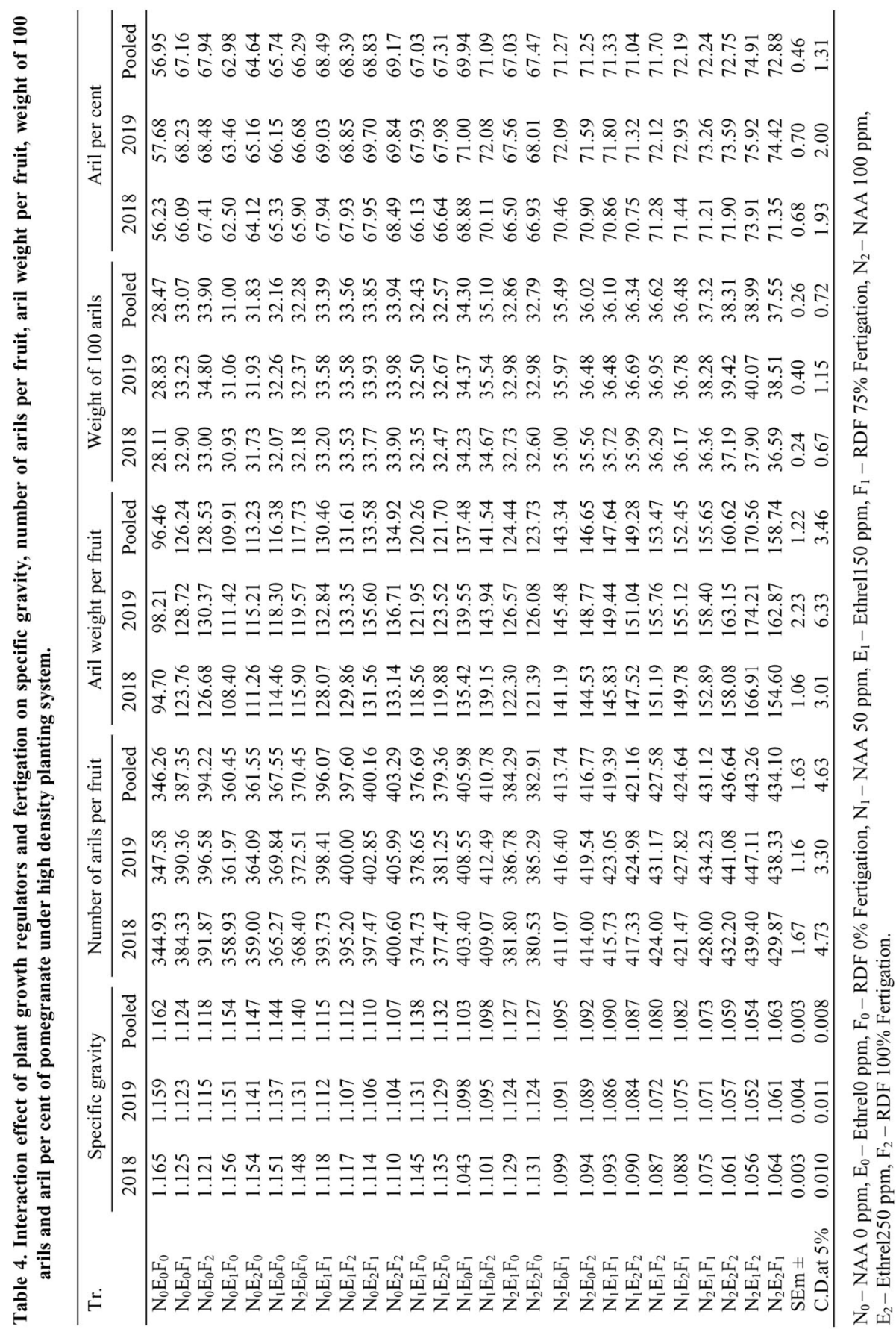


Table 5. Interaction effect of plant growth regulators and fertigation on rind weight (g), rind thickness (mm) rind per cent and fruit cracking per cent of pomegranate under high density planting system.

\begin{tabular}{|c|c|c|c|c|c|c|c|c|c|c|c|c|}
\hline \multirow[t]{2}{*}{ Tr. } & \multicolumn{3}{|c|}{ Rind weight (g) } & \multicolumn{3}{|c|}{ Rind thickness (mm) } & \multicolumn{3}{|c|}{ Rind per cent } & \multicolumn{3}{|c|}{ Fruit cracking per cent } \\
\hline & 2018 & 2019 & Pooled & 2018 & 2019 & Pooled & 2018 & 2019 & Pooled & 2018 & 2019 & Pooled \\
\hline $\mathrm{N}_{0} \mathrm{E}_{0} \mathrm{~F}_{0}$ & 73.55 & 72.10 & 72.83 & 6.66 & 5.87 & 6.27 & 43.78 & 42.32 & 43.05 & 7.53 & 6.97 & 7.25 \\
\hline $\mathrm{N}_{0} \mathrm{E}_{0} \mathrm{~F}_{1}$ & 63.51 & 59.98 & 61.74 & 5.69 & 4.94 & 5.32 & 33.91 & 31.77 & 32.84 & 6.37 & 5.53 & 5.95 \\
\hline $\mathrm{N}_{0} \mathrm{E}_{0} \mathrm{~F}_{2}$ & 61.27 & 60.07 & 60.67 & 5.68 & 4.92 & 5.30 & 32.59 & 31.52 & 32.06 & 6.33 & 5.47 & 5.90 \\
\hline $\mathrm{N}_{0} \mathrm{E}_{1} \mathrm{~F}_{0}$ & 64.87 & 64.22 & 64.55 & 5.83 & 5.13 & 5.48 & 37.50 & 36.54 & 37.02 & 7.10 & 6.30 & 6.70 \\
\hline $\mathrm{N}_{0} \mathrm{E}_{2} \mathrm{~F}_{0}$ & 62.30 & 61.60 & 61.95 & 5.82 & 5.09 & 5.46 & 35.88 & 34.84 & 35.36 & 6.93 & 6.10 & 6.52 \\
\hline $\mathrm{N}_{1} \mathrm{E}_{0} \mathrm{~F}_{0}$ & 60.77 & 60.57 & 60.67 & 5.82 & 5.08 & 5.45 & 34.67 & 33.85 & 34.26 & 6.87 & 6.07 & 6.47 \\
\hline $\mathrm{N}_{2} \mathrm{E}_{0} \mathrm{~F}_{0}$ & 59.99 & 59.75 & 59.87 & 5.80 & 5.05 & 5.43 & 34.10 & 33.32 & 33.71 & 6.83 & 6.00 & 6.42 \\
\hline $\mathrm{N}_{0} \mathrm{E}_{1} \mathrm{~F}_{1}$ & 60.49 & 59.63 & 60.06 & 5.67 & 4.89 & 5.28 & 32.06 & 30.97 & 31.52 & 6.23 & 5.40 & 5.82 \\
\hline $\mathrm{N}_{0} \mathrm{E}_{1} \mathrm{~F}_{2}$ & 61.32 & 60.31 & 60.82 & 5.67 & 4.89 & 5.28 & 32.07 & 31.15 & 31.61 & 6.20 & 5.40 & 5.80 \\
\hline $\mathrm{N}_{0} \mathrm{E}_{2} \mathrm{~F}_{1}$ & 62.04 & 59.02 & 60.53 & 5.64 & 4.88 & 5.26 & 32.05 & 30.30 & 31.17 & 6.17 & 5.33 & 5.75 \\
\hline $\mathrm{N}_{0} \mathrm{E}_{2} \mathrm{~F}_{2}$ & 61.44 & 59.15 & 60.30 & 5.63 & 4.84 & 5.24 & 31.51 & 30.16 & 30.83 & 6.13 & 5.30 & 5.72 \\
\hline $\mathrm{N}_{1} \mathrm{E}_{1} \mathrm{~F}_{0}$ & 60.72 & 57.72 & 59.22 & 5.78 & 5.06 & 5.42 & 33.87 & 32.07 & 32.97 & 6.60 & 5.87 & 6.23 \\
\hline $\mathrm{N}_{1} \mathrm{E}_{2} \mathrm{~F}_{0}$ & 60.04 & 58.17 & 59.11 & 5.77 & 5.03 & 5.40 & 33.36 & 32.02 & 32.69 & 6.50 & 5.80 & 6.15 \\
\hline $\mathrm{N}_{1} \mathrm{E}_{0} \mathrm{~F}_{1}$ & 61.15 & 56.80 & 58.97 & 5.59 & 4.83 & 5.21 & 31.12 & 28.83 & 30.06 & 6.10 & 5.30 & 5.70 \\
\hline $\mathrm{N}_{1} \mathrm{E}_{0} \mathrm{~F}_{2}$ & 59.73 & 55.70 & 57.72 & 5.58 & 4.83 & 5.20 & 29.89 & 27.92 & 28.91 & 6.10 & 5.27 & 5.68 \\
\hline $\mathrm{N}_{2} \mathrm{E}_{1} \mathrm{~F}_{0}$ & 61.66 & 60.87 & 61.27 & 5.72 & 4.98 & 5.35 & 33.49 & 32.44 & 32.96 & 6.40 & 5.57 & 5.98 \\
\hline $\mathrm{N}_{2} \mathrm{E}_{2} \mathrm{~F}_{0}$ & 60.21 & 59.31 & 59.76 & 5.75 & 5.00 & 5.38 & 33.07 & 31.99 & 32.53 & 6.47 & 5.70 & 6.08 \\
\hline $\mathrm{N}_{2} \mathrm{E}_{0} \mathrm{~F}_{1}$ & 59.35 & 56.32 & 57.83 & 5.55 & 4.81 & 5.18 & 29.54 & 27.91 & 28.73 & 6.10 & 5.23 & 5.67 \\
\hline $\mathrm{N}_{2} \mathrm{E}_{0} \mathrm{~F}_{2}$ & 59.35 & 59.12 & 59.24 & 5.52 & 4.79 & 5.15 & 29.10 & 28.41 & 28.75 & 6.07 & 5.20 & 5.63 \\
\hline $\mathrm{N}_{1} \mathrm{E}_{1} \mathrm{~F}_{1}$ & 60.04 & 58.68 & 59.36 & 5.52 & 4.76 & 5.14 & 29.14 & 28.20 & 28.67 & 6.00 & 5.20 & 5.60 \\
\hline $\mathrm{N}_{1} \mathrm{E}_{2} \mathrm{~F}_{2}$ & 61.05 & 60.80 & 60.93 & 5.48 & 4.75 & 5.11 & 29.25 & 28.68 & 28.96 & 5.97 & 5.17 & 5.57 \\
\hline $\mathrm{N}_{1} \mathrm{E}_{1} \mathrm{~F}_{2}$ & 61.02 & 60.08 & 60.55 & 5.42 & 4.72 & 5.07 & 28.72 & 27.88 & 28.30 & 5.90 & 5.10 & 5.50 \\
\hline $\mathrm{N}_{1} \mathrm{E}_{2} \mathrm{~F}_{1}$ & 59.87 & 57.73 & 58.80 & 5.46 & 4.72 & 5.09 & 28.56 & 27.07 & 27.81 & 5.97 & 5.13 & 5.55 \\
\hline $\mathrm{N}_{2} \mathrm{E}_{1} \mathrm{~F}_{1}$ & 61.83 & 57.88 & 59.86 & 5.41 & 4.70 & 5.05 & 28.80 & 26.74 & 27.77 & 5.90 & 5.07 & 5.48 \\
\hline $\mathrm{N}_{2} \mathrm{E}_{2} \mathrm{~F}_{2}$ & 61.81 & 58.55 & 60.18 & 5.39 & 4.64 & 5.02 & 28.10 & 26.41 & 27.25 & 5.80 & 4.87 & 5.33 \\
\hline $\mathrm{N}_{2} \mathrm{E}_{1} \mathrm{~F}_{2}$ & 59.05 & 54.96 & 57.00 & 5.34 & 4.60 & 4.97 & 26.09 & 24.08 & 25.09 & 5.70 & 4.53 & 5.12 \\
\hline $\mathrm{N}_{2} \mathrm{E}_{2} \mathrm{~F}_{1}$ & 62.05 & 56.13 & 59.09 & 5.41 & 4.67 & 5.04 & 28.65 & 25.58 & 27.11 & 5.87 & 5.03 & 5.45 \\
\hline $\mathrm{SEm} \pm$ & 0.79 & 0.93 & 0.59 & 0.07 & 0.06 & 0.05 & 0.65 & 0.72 & 0.48 & 0.03 & 0.09 & 0.06 \\
\hline $\begin{array}{c}\text { C.D.at } \\
5 \%\end{array}$ & 2.24 & 2.64 & 1.66 & 0.21 & 0.18 & 0.14 & 1.84 & 2.06 & 1.36 & 0.10 & 0.26 & 0.17 \\
\hline
\end{tabular}

$\mathrm{N}_{0}$ - NAA 0 ppm, $\mathrm{E}_{0}-$ Ethrel 0 ppm, $\mathrm{F}_{0}-\mathrm{RDF} 0 \%$ Fertigation, $\mathrm{N}_{1}-\mathrm{NAA} 50 \mathrm{ppm}, \mathrm{E}_{1}-$ Ethrel $150 \mathrm{ppm}, \mathrm{F}_{1}-\mathrm{RDF} 75 \%$ Fertigation, $\mathrm{N}_{2}-\mathrm{NAA} 100 \mathrm{ppm}, \mathrm{E}_{2}-$ Ethrel 250 ppm, $\mathrm{F}_{2}-$ RDF $100 \%$ Fertigation.

It is evident from the data presented in Tables 1 and 3 that NAA, ethrel and fertigation levels had significant effect on the fruit quality attributes. NAA $\left(100 \mathrm{ppmN}_{2}\right)$ showed minimum mean specific gravity (1.092), rind weight $(59.34 \mathrm{~g})$, rind thickness $(5.17 \mathrm{~mm})$, rind per cent $(29.32 \%)$ and fruit cracking per cent $(5.69 \%)$. Similarly, ethrel $\left(250 \mathrm{ppm}, \mathrm{E}_{2}\right)$ had significant effect on the fruit quality attributes and was found to be minimum mean specific gravity (1.102), rind weight $(60.07 \mathrm{~g})$, rind thickness $(5.22 \mathrm{~mm})$, rind per cent $(30.41 \%)$ and fruit cracking per cent $(5.79 \%)$. Among the different levels of fertigation, minimum mean specific gravity (1.090), rind thickness $(5.15 \mathrm{~mm})$, rind per cent $(29.09 \%)$ and fruit cracking $(5.58 \%)$ were recorded in the treatment fertigation 100 per cent $\operatorname{RDF}\left(\mathrm{F}_{2}\right)$. However, minimum mean rind weight was observed in treatment $F_{1}(59.58 \mathrm{~g})$ which consists of 75 per cent RDF. The interaction effect of NAA, ethrel and fertigation (Tables 4 and 5) was found better than their individual effect. Minimum mean 
specific gravity (1.054), rind weight $(57.00 \mathrm{~g})$, rind thickness $(4.97 \mathrm{~mm})$, rind per cent $(25.09 \%)$ and fruit cracking per cent $\left(5.12 \%\right.$ ) was recorded in $\mathrm{N}_{2} \mathrm{E}_{1} \mathrm{~F}_{2}$ (NAA $100 \mathrm{ppm}+$ ethrel $150 \mathrm{ppm}+$ fertigation 100\% RDF). This result is in agreement with the finding of Tsomu and Patel (2019). Minimum values observed for this quality with fertigation treatments might be due to higher fertilizer use efficiency as well as uptake of nutrients. Further, reduction in specific gravity of fruit by the application of NAA might be due to accumulation of more metabolites resulting higher weight at faster rate than increase in the volume of fruits. More moisture induced the thinner rind which is one of the desirable characters for fruit. As rind thickness reduced due to fruit development of aril growth which was influenced as a result of various fertigation recorded in this investigation. The treatment combination which exhibited the thinner rind might be on account of more development of fruits in general and aril in particular. Regarding the rind percentage, 100\% WSF fertigation treatment combinations showed the lowest rind percentage than control. Similar decrease in rind weight as a result of fertilizers was also recorded by Chougule (1976) in pomegranate. The present results are also in accordance with the results of Dalal et al. (2018) in acid lime. The reduction in rind weight, rind thickness and rind percentage due to NAA and ethrel application might be attributed to increase in cell wall plasticity which caused cell enlargement, thus stretched the rind and made it thinner (Thakur and Sharma 2018). Similarly reduced peel weight by spray of NAA and ethrel were also found by Harikanth et al. (2018) in grape. However, lower fruit cracking per cent was also observed under fertigation treatment. The moisture stress affects the fruit development and results in fruit cracking. Pomegranate being drought resistance crop, use of drip fertigation was advantageous, as fruit cracking was reduced due to less moisture stress. Rani and Brahmachari (2001) recorded that the spray of NAA application on litchi resulted in least fruit cracking. Similarly, Kumar et al.(2017) also found that spray of NAA significantly lower the incidence of cracking in pomegranate.

Based on the overall effects of the different treatments it may be concluded that the individual effect of different plant growth regulating substances and fertigation was found significant but not much affected the physical quality of pomegranate but in interaction effects physical quality parameters was significantly better under $\mathrm{N}_{2} \mathrm{E}_{1} \mathrm{~F}_{2}$ (NAA @ 100 ppm + ethrel @ 150 ppm + fertigation@100\% RDF). Hence treatment, $\mathrm{N}_{2} \mathrm{E}_{1} \mathrm{~F}_{2}$ may be considered worth for application in pomegranate for better harvest of the crop.

\section{References}

Bankar GJ,Dass HC, Prasad, RN and Vashishtha BB 1990. Nitrogen nutrition in pomegranate under arid conditions. Ann. Arid Zone 29: 63-66.

Chougule SB 1976. Effect of nitrogen, phosphorus and potassium on growth yield and quality of pomegranate (Punica granatum L.). M.Sc. Thesis, submitted to GAU, Ahmadabad.

Dalal NR,Pawar PS and TaruAS 2018. Studies on irrigation, fertigation and their interaction on yield and quality parameters of acid lime. J. Multilo. Sci. 8(26): 22-26.

Gaikwad PS, Tambe TB and Gaonkar YA 2019. Effect of foliar application of chemicals on yield of pomegranate cv. Bhagwa. Int. J. Chem. Stud. 7(1): 524-528.

Harikanth P, Kumar PS,Satisha J, Upreti KKand Kumar M 2018. Effect of good viticultural practices to reduce bunch compactness and berry size improvement in grapes cv. 'Crimson Seedless.' Int. J. Chem. Stud. 6(3):1061-1066.

Kumar K, Pinder R, Jitender STK, Dabas S, Yadav B and Rana S 2017. Effect of growth regulators and micronutrients on fruit cracking and fruit yield in pomegranate. Indian J. Agric. Res. 51(3): 272-276.

Mikal ES 1999. Postha. Bio. and Tech.15: 279-292.

Pareek OP 1982. Pomegranate research report and appraisal. Tech. Doc. No.8, AICFIP on arid zone fruits. 
Rani R and Brahmachari VS 2001. Effect of plant bioregulators on cracking and physico-chemical composition of fruit in china litchi. Prog. Hort. 33(1): 32-36.

Shanmugasundaram T and Balakrishnamurthy G2017. Exploitation of plant growth substances for improving the yield and quality of pomegranate under ultra high density planting. Int. J. Curr. Microbiol. App. Sci. 6(3): 102-109.

Sinha R, Karuna K, Prasad M, Singh G,Mankar A and Ahmad F2019. Growth and yield of Kinnow mandarin in response to fertigation. Int. J. Chem. Stud. 7(3): 2602-2605.

Thakur C and Sharma CL 2018. Effect of plant growth regulators on physico-chemical parameters of pomegranate (Punica granatum L.) cv. Kandhari. Int. J. Chem. Stud. 6(4): 1849-1855.

Thiyagarajan T 2006. Effect of micro sprinkler on growth, yield and nutrient dynamics pattern in groundnut. Ph.D. (Engineering) Thesis, Tamil Nadu Agri. Univ. Coimbatore.

Tsomu T and Patel HC2019. Influence of plant growth regulators and micronutrients on yield and quality attributes of mango cv. Mallika. J. Pharmacogn. Phytochem. 8(4): 1240-1247.

(Manuscript received on 5 June, 2020; revised on 29 March, 2021) 